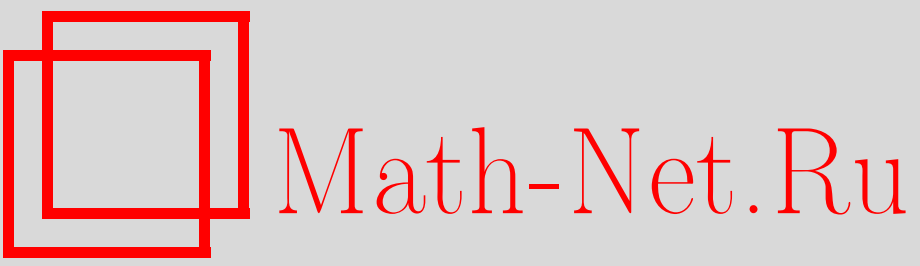

Ю. В. Брежнев, Конечнозонные потенциалы с тригональными кривыми, ТМФ, 2002, том 133, номер 3, 398-404

DOI: https://doi.org/10.4213/tmf406

Использование Общероссийского математического портала Math-Net.Ru подразумевает, что вы прочитали и согласны с пользовательским соглашением

http://www.mathnet.ru/rus/agreement

Параметры загрузки:

IP : 54.162 .127 .20

26 апреля 2023 г., 12:10:22 
ТЕОРЕТИЧЕСКАЯ

И МАТЕМАТИЧЕСКАЯ

ФИЗИКА

Том 133, № 3

декабрь, 2002

(C) 2002 г.

\author{
Ю.В. Брежнев*
}

\title{
КОНЕЧНОЗОННЫЕ ПОТЕНЦИАЛЫ С ТРИГОНАЛЬНЫМИ КРИВЫМИ
}

Для конечнозонных операторов с тригональными кривыми построены уравнения Дубровина и формулы следов. Приведены примеры.

Ключевые слова: конечнозонные потенциалы, спектральные задачи, формулы следов.

\section{1. ВВЕДЕНИЕ}

Теория конечнозонного интегрирования для оператора Шредингера достаточно хорошо развита. Для этой задачи в 70-е годы были разработаны почти все атрибуты алгебро-геометрического интегрирования: спектр и монодромия [1], [2], $\Psi$-функция [2], [3] и абелевы многообразия [4], уравнения на нули и полюсы $\Psi$-функции [5] как аналоги данных рассеяния и восстановление потенциала по ним в периодическом случае [5], [6]. Позднее многие из этих результатов были получены и для других уравнений (для уравнения синус-Гордон, модифицированного уравнения Кортевега-де Фриза (КдФ), нелинейного уравнения Шредингера) и матричных операторов [6]. В конце 80-х и в 90-х годах были проведены многочисленные исследования проблемы эффективизации общих $\Theta$-функциональных формул и вешественности решений [7], [8] и, что важно с прикладной точки зрения, редукции к эллиптическим решениям [9]. Библиография по этой тематике обширна, мы укажем лишь недавний обзор [10] и готовяшуюся монографию [11].

В то же время для ряда нелинейных уравнений и соответствующих им спектральных задач не разработаны или даже отсутствуют некоторые составляюшие алгебро-геометрического интегрирования. Это касается уравнений Буссинеска и Цицейки, системы спаренных уравнений Шредингера (системы Манакова) и вообше уравнений с размерностью $L-A$-пар, больше или равной трем. Многие из этих уравнений имеют важные физические приложения.

Причины такого отсутствия как чисто технические, связанные с повышением порядка спектральной задачи, так и более сушественные - переход от гиперэллиптических к более общим алгебраическим кривым. Например, для иерархии уравнения Буссинеска,

* Калининградский государственный университет, Калининград, Россия. E-mail: brezhnev@mail.ru 
а точнее связанной с ним спектральной задачи

$$
\Psi^{\prime \prime \prime}+u(x) \Psi^{\prime}+v(x) \Psi=\lambda \Psi
$$

формализм стационарных уравнений и полиномиальная $\lambda$-техника описаны сравнительно недавно [12]. Сказанное выше относится и к решениям из области эллиптических солитонов [9]. Что касается явной временно́й динамики, то таких решений еше меньше [13], [14].

Как известно, еще в работе Новикова [1] было отмечено, что стационарные уравнения КдФ (уравнения Новикова) являются конечномерными гамильтоновыми (в переменных Остроградского $u, u_{x}, \ldots$ [15]) системами, интегрируемыми по Лиувиллю. Окончательные выражения динамики по $x$ и $t$ в виде линейных фаз $k x+c t$ дают, как известно, знаменитые тождества следов, выражающие потенциал спектральной задачи через координаты $\gamma_{k}(x, t)$ нулей $\Psi$-функции. Эволюция величин $\gamma_{k}$ описывается системой нелинейных обыкновенных автономных дифференциальных уравнений - уравнениями Дубровина (УД), которые после преобразования Абеля сводятся к обрашению абелевых интегралов первого рода (задача Якоби).

Гельфанд и Дикий показали [16], что для уравнения КдФ в процедуре интегрирования по Лиувиллю переход от переменных Остроградского к каноническим переменным фактически может быть стандартизирован на уровне полиномиальной техники. С другой стороны, собственно в теории конечномерных гамильтоновых систем УД чаще возникают уже в виде спектральной (алгебраической) кривой и задачи обрашения Якоби для нее. Связь этих объектов с явным представлением потенциала как симметрической функции с линейной зависимостью от $x, t$ точки на якобиане кривой дает формулы следов.

Однако применительно к спектральным задачам порядка выше двух или к общим операторным $\lambda$-пучкам указанные конструкции не разрабатывались. Отметим задачу Ковалевской и ее эллиптические решения [17] как наиболее показательный пример, в котором формулы следов и УД в форме задачи обрашения Якоби одинаково необходимы. Недавно были опубликованы примеры более непосредственной связи упомянутых атрибутов со стационарными уравнениями Буссинеска и негиперэллиптическими кривыми [18].

В данной работе показано, что УД и формулы следов сушествуют и в тригональном случае. Из приведенных примеров очевидно, что $n$-гональные обобщения (иерархия Гельфанда-Дикого) тоже имеют место. Схема решения следует из построений.

\section{2. Ч-ФУНКЦИЯ И УРАВНЕНИЯ ДУБРОВИНА}

Воспользуемся двумя ключевыми моментами, анонсированными в работах [19], [20]: фундаментальным свойством конечнозонных операторов и УД на общие операторные пучки. Пусть потенциал $(u, v)$ в задаче $(1)$ конечнозонный. Мы не обсуждаем здесь различия между устоявшимся термином спектральной теории операторов "конечнозонньй (конечно-лакунный) потенциал" и термином "алгебро-геометрический потенциал". 
Критерием конечнозонности может выступать наличие парного коммутирующего оператора [3]. Поскольку коммутация подразумевается на решениях уравнения (1), то сразу отождествим коммутирующий оператор с $\lambda$-пучком. Обозначим его собственное значение как $\mu$ :

$$
A(\lambda ; x) \Psi^{\prime \prime}+B(\lambda ; x) \Psi^{\prime}+C(\lambda ; x) \Psi=\mu \Psi
$$

где

$$
C=\frac{1}{3} A^{\prime \prime}-B^{\prime}+\frac{2}{3} u A .
$$

Выражение для $C(\lambda ; x)$ следует из совместности (2) и (1). Полиномы $A$ и $B$ определяют иерархию.

ЛЕмма 1. Конечнозонная $\Psi$-функиия имеет вид ${ }^{1)}$

$$
\frac{\Psi^{\prime}}{\Psi}=\frac{\left(A^{\prime}+B\right)(\mu-C)-v A^{2}+A C^{\prime}}{3 A \mu+A A^{\prime \prime}+u A^{2}+3 B\left(A^{\prime}+B\right)} .
$$

ДОКАЗАТЕЛЬСТВО сводится к последовательному исключению производных $\Psi$ в уравнениях (1) и (2).

ЛЕмма 2. Алгебраическая кривая, соответствующая $\Psi$-функиии (3), имеет вuд $\partial^{2)}$

$$
\mu^{3}+\frac{1}{3}\left(A A^{(\mathrm{IV})}+\left(2 A^{\prime}+3 B\right) A^{\prime \prime \prime}+3 A B^{\prime \prime \prime}-A^{\prime \prime 2}-\cdots\right) \mu+(\ldots)=0
$$

Формула (4) получается подстановкой (3) в (1). Рекуррентные формулы для полиномов (по $\lambda) A, B$ приведены в работе [12], там же имеются леммы 1 и 2 и нижеследующее предложение 1 , хотя и без доказательств и в довольно громоздких обозначениях. Определим $\gamma_{k}(x)$ как нули $\Psi$-функции, зависящие от $\lambda$. Пусть $\mu_{k}(x)$ - корни уравнения (4) при $\lambda=\gamma_{k}(x)$, записанного в виде

$$
\mu_{k}^{3}+Q\left(\gamma_{k}\right) \mu_{k}+R\left(\gamma_{k}\right)=0
$$

ПрЕДЛОЖЕНИЕ 1. Дивизор $\left(\gamma_{k}, \mu_{k}\right)$ нулей $\Psi$-функиии удовлетворяет системе уравнений, записанной в форме дифференциалов:

$$
\begin{aligned}
\frac{d \gamma_{k}}{3 \mu_{k}^{2}+Q\left(\gamma_{k}\right)} & =\frac{3 A\left(\gamma_{k} ; x\right)}{a \prod_{j \neq k}\left(\gamma_{k}-\gamma_{j}\right)} d x, \\
\frac{d \mu_{k}}{Q^{\prime}\left(\gamma_{k}\right) \mu_{k}+R^{\prime}\left(\gamma_{k}\right)} & =-\frac{3 A\left(\gamma_{k} ; x\right)}{a \prod_{j \neq k}\left(\gamma_{k}-\gamma_{j}\right)} d x
\end{aligned}
$$

\footnotetext{
1) Мы не пишем очевидную экспоненту от интеграла, так как далее нам понадобится формула в виде (3).

${ }^{2)}$ Многоточием в скобках обозначены оставшиеся слагаемые с низшими производными от $A, B$.
} 
ДокАЗАтЕльство. Обрашаем в полином правую часть (3) как рациональную функцию по $\mu$ в силу уравнения кривой (4). В результате получаем формулу

$$
\frac{\Psi^{\prime}}{\Psi}=\frac{3 A \mu^{2}-\left(A A^{\prime \prime}+u A^{2}+3 B^{2}+3 B A^{\prime}\right) \mu+\cdots}{\left(3 v-u^{\prime}\right) A^{3}+\left(3 B u+2 A^{\prime} u+2 A^{\prime \prime \prime}+3 B^{\prime \prime}\right) A^{2}+\cdots} .
$$

Обозначим знаменатель как

$$
\Pi(\lambda ; x)=\left(3 v-u^{\prime}\right) A^{3}+\left(3 B u+2 A^{\prime} u+2 A^{\prime \prime \prime}+3 B^{\prime \prime}\right) A^{2}+3\left(A^{\prime \prime}+B^{\prime}\right) B A+3 B^{2}\left(A^{\prime}+B\right)
$$

Все нули функции $\Psi$ являются полюсами для $\Psi^{\prime} / \Psi$. Определим их, факторизуя П:

$$
\Pi(\lambda ; x)=a\left(\lambda-\gamma_{1}\right) \ldots\left(\lambda-\gamma_{g}\right),
$$

где $a$ - числовая константа. Используя (3), при $\lambda \rightarrow \gamma_{k}$ получаем важное соотношениевторую координату нуля:

$$
\mu_{k}=\frac{F\left(\gamma_{k} ; x\right)}{A\left(\gamma_{k} ; x\right)}
$$

где введено обозначение

$$
-3 F=A^{\prime \prime}+u A^{2}+3 B\left(A^{\prime}+B\right) .
$$

В результате имеем

$$
\frac{\Psi^{\prime}}{\Psi}=\frac{\left(A^{\prime}+B\right)(\mu-C)+A C^{\prime}-v A^{2}}{A \mu-F}=-3 \frac{\left(\mu^{2}+Q\right) A^{2}+A F \mu+F^{2}}{A \Pi}+\frac{A^{\prime}+B}{A} .
$$

В пределе $\lambda \rightarrow \gamma_{k}$ с учетом (11) приходим к формуле (6). Формула (7) получается дифференцированием уравнения (5) с использованием соотношения (6).

\section{3. ЦЕНТРАЛЬНАЯ ФОРМУЛА СЛЕДОВ И ИНТЕГРИРОВАНИЕ УРАВНЕНИЙ ДУБРОВИНА}

Тригональные кривые проявляют бо́льшую нестандартность, чем гиперэллиптические, что проявляется на структуре УД. В самом деле, УД (6), (7) содержат потенциал в функции $A$ и поэтому в такой форме теряют смысл. Формулы следов становятся, таким образом, неизбежным атрибутом придания УД автономной формы с последующим интегрированием. В свою очередь, потенциал $(u, v)$, как абелева функция, будет симметричной комбинацией, вообще говоря, обеих координат $\left(\gamma_{k}, \mu_{k}\right)$ :

$$
u(x)=S_{1}\left(\gamma_{k}, \mu_{k}\right), \quad v(x)=S_{2}\left(\gamma_{k}, \mu_{k}\right) .
$$

Высшие производные будут выражаться через $\left(\gamma_{k}, \mu_{k}\right)$ в силу самих УД (6), (7) и (12). По этой причине мы будем называть соотношения (12) центральными формулами следов. Другие тождества следов становятся их следствиями. Общая техника вывода этих формул, по всей видимости, должна использовать теорию симметрических функций от двух переменных. Здесь мы ограничимся характерными примерами.

3 Теоретическая и математическая физика, т. 133, № 3, 2002 г. 


\section{4. ПРИМЕРЫ}

Приводя примеры, мы будем исходить из спектральной задачи (1), не комментируя связанные с ней нелинейные уравнения, так как они хорошо известны. Это уравнения Савади-Котера и Буссинеска. Заметим, что все они укладываются в обшую иерархию уравнения Буссинеска [12], но с различными редукциями на потенциал $(u, v)$. Из стационарной иерархии хорошо известных $L-A$-пар для этих уравнений мы получаем и коммутируюшие операторные пучки.

ПримеР 1. $v=0$. Коммутируюший пучок имеет вид

$$
\left(-9 \lambda+3 u^{\prime}\right) \Psi^{\prime \prime}+\left(u^{2}-u^{\prime \prime}+c\right) \Psi^{\prime}-6 \lambda u \Psi=\mu \Psi .
$$

Здесь и далее через $c$ мы обозначаем первую константу $c_{j}$, нумеруюшую члены иерархии. Получаем далее

$$
A\left(\gamma_{k} ; x\right)=-9 \gamma_{k}+3 u^{\prime}
$$

Полином (9) приобретает вид

$$
3^{-7} \Pi(\lambda ; x)=\lambda^{4}-\frac{2}{3} u^{\prime} \lambda^{3}+\frac{1}{9}\left(u^{(\mathrm{IV})}+3 u u^{\prime \prime}+2 u^{\prime 2}+u^{3}+c u\right) \lambda^{2}+(\ldots) .
$$

Используя (10) и эту формулу, получаем одно из тождеств следов:

$$
u^{\prime}=\frac{2}{3} \sum_{j=1}^{4} \gamma_{j},
$$

которое сразу приводит УД (6), (7) к автономной форме

$$
\frac{2 \cdot 81 d \gamma_{k}}{3 \mu_{k}^{2}+Q\left(\gamma_{k}\right)}=\frac{\sum_{j=1}^{4} \gamma_{j}-2 \gamma_{k}}{\prod_{j \neq k}\left(\gamma_{k}-\gamma_{j}\right)} d x
$$

Выпишем формулу для кривой (4):

$$
\mu^{3}+\left(27 c \lambda^{2}+E_{1}\right) \mu+729 \lambda^{5}+81 E_{2} \lambda^{3}+E_{3} \lambda=0,
$$

где $E_{j}$ - дифференциальные полиномы по $u(x)$. Имеет место следуюшее

ПрЕДЛОЖенИЕ 2. Уравнения (14) эквивалентны задаче обращения Якоби для кривой (15)

$$
\begin{gathered}
\sum_{k=1}^{4} \int^{\left(\gamma_{k}, \mu_{k}\right)} \frac{d \lambda}{3 \mu^{2}+Q(\lambda)}=a_{1}, \quad \sum_{k=1}^{4} \int^{\left(\gamma_{k}, \mu_{k}\right)} \frac{\lambda d \lambda}{3 \mu^{2}+Q(\lambda)}=a_{2}, \\
\sum_{k=1}^{4} \int^{\left(\gamma_{k}, \mu_{k}\right)} \frac{\mu d \lambda}{3 \mu^{2}+Q(\lambda)}=a_{3}, \quad \sum_{k=1}^{4} \int^{\left(\gamma_{k}, \mu_{k}\right)} \frac{\lambda^{2} d \lambda}{3 \mu^{2}+Q(\lambda)}=a_{4}-\frac{1}{81} x \\
Q(\lambda)=27 c \lambda^{2}+E_{1} .
\end{gathered}
$$

Центральная формула следов (12) имеет вид

$$
u=\frac{1}{6} \sum_{k=1}^{4} \gamma_{k} \mu_{k} \frac{\sum_{j=1}^{4} \gamma_{j}-2 \gamma_{k}}{\prod_{j \neq k}\left(\gamma_{k}-\gamma_{j}\right)} .
$$


ДокАЗАТЕльСтво. Первое, второе и четвертое соотношения в (16) проверяются непосредственным домножением правых частей (14) на единицу, $\gamma_{k}$ и $\gamma_{k}^{2}$, соответственно, с последуюшим суммированием и интегрированием. Таким образом, они являются простыми тождествами в теории симметрических функций по переменным $\gamma_{k}$. Для доказательства третьего равенства в (16) необходимо воспользоваться формулой для второй координаты (11)

$$
\begin{aligned}
\mu_{k}= & \frac{1}{3 u^{\prime}-9 \gamma_{k}}\left\{27 u \gamma_{k}^{2}-9\left(u^{\prime \prime \prime}+2 u u^{\prime}\right) \gamma_{k}+3 u^{\prime \prime \prime} u^{\prime}-2 u^{\prime \prime 2}+\right. \\
& \left.+\left(c+u^{2}\right)\left(u^{\prime \prime}+u^{2}+c\right)+3 u u^{\prime 2}\right\} .
\end{aligned}
$$

Подставляя (18) в сумму

$$
\sum_{k=1}^{4} \frac{\mu_{k} d \gamma_{k}}{3 \mu_{k}+Q\left(\gamma_{k}\right)}
$$

убеждаемся, что она равна выражению

$$
3 \frac{\left(u^{2}+2 u^{\prime \prime}+c\right)\left(u^{2}-u^{\prime \prime}+c\right)\left(3 \sum_{k=1}^{4} \gamma_{k}-2 u^{\prime}\right)}{\prod_{k=1}^{4}\left(3 \gamma_{k}-u^{\prime}\right)},
$$

которое в силу (13) равно нулю. Домножая правую часть (14) на $\gamma_{k} \mu_{k}$, суммируя и используя (18), получаем формулу (17).

ПримеР 2. Спектральная задача (1) с коммутирующим пучком вида

$$
u \Psi^{\prime \prime \prime}+\left(3 \lambda+v-u^{\prime}+c\right) \Psi^{\prime}+\left(\frac{2}{3} u^{\prime \prime}-v^{\prime}+\frac{2}{3} u^{2}\right) \Psi=\mu \Psi
$$

Этот пример более показателен, так как здесь мы имеем два потенциала $u$ и $v$ :

$$
A=u, \quad B=3 \lambda+v-u^{\prime}+c
$$

род $g=3$. Факторизуя П $(\lambda ; x)$ (числовая константа $a=81)$ :

$$
\begin{aligned}
3^{-4} \Pi(\lambda ; x)= & \lambda^{3}+\left(v+c-\frac{2}{3} u^{\prime}\right) \lambda^{2}+ \\
& +\frac{1}{9}\left(\frac{2}{3} u^{3}+u v^{\prime}+u^{\prime 2}+3(v+c)^{2}-4(v+c) u^{\prime}\right) \lambda+(\ldots),
\end{aligned}
$$

получаем тождество следов $v+c-2 u^{\prime} / 3=-\gamma_{1}-\gamma_{2}-\gamma_{3}$, из которого можно считать $v$ найденным, если будет найдено $u$ (а следовательно, и $\left.u^{\prime}\right)$. Найдем его. Уравнения (6), (7) и вторая координата (11) имеют вид

$$
\begin{gathered}
\gamma_{k}^{\prime}=\frac{3 \mu_{k}^{2}+Q\left(\gamma_{k}\right)}{27 \prod_{j \neq k}\left(\gamma_{k}-\gamma_{j}\right)} \\
\mu_{k}=\frac{27 \gamma_{k}^{2}+9\left(2 v-u^{\prime}+2 c\right) \gamma_{k}+u u^{\prime \prime}+u^{3}+3(c+v)\left(c+v-3 u^{\prime}\right)}{3 u}
\end{gathered}
$$


Кривая (4) и задача обрашения Якоби выписьваются с очевидностью, и мы их не приводим. Используя последнюю формулу, находим однозначно $u, v$ :

$$
\frac{9}{u}=-\sum_{k=1}^{3} \frac{\mu_{k}}{\prod_{j \neq k}\left(\gamma_{k}-\gamma_{j}\right)}, \quad v=\frac{2}{3} u^{\prime}-c-\sum_{k=1}^{3} \gamma_{k}
$$

Нам не удалось придать компактный вид формуле для $v$ без $u$ через $\lambda, \mu$.

Благодарности. Автор выражает признательность проф. К. Эйлбеку и В. Энольскому за многочисленные обсуждения. Работа выполнена при финансовой поддержке РФФИ (грант № 00-01-00782) и Королевского общества Великобритании.

\section{Список литературы}

[1] С. П. Новиков. Функц. анализ и его прилож. 1974. Т. 8. № 3. С. 54-66.

[2] А. Р. Итс, В. Б. Матвеев. Функц. анализ и его прилож. 1975. Т. 9. № 1. С. 69-70.

[3] И. М. Кричевер. Функц. анализ и его прилож. 1976. Т. 10. № 2. С. 75-76.

[4] Б. А. Дубровин. Функц. анализ и его прилож. 1977. Т. 11. № 4. С. 28-41.

[5] Б. А. Дубровин. Функц. анализ и его прилож. 1975. Т. 9. № 3. С. 41-51.

[6] Б. А. Дубровин, В. Б. Матвеев, С. П. Новиков. УМН. 1976. Т. 31. № 1. С. 55-136.

[7] Б. А. Дубровин, С. М. Натанзон. Функц. анализ и его прилож. 1982. Т. 16. № 1. С. $27-43$.

[8] С. П. Новиков. Зап. научн. семин. ЛОМИ. 1984. Т. 133. С. 177-196.

[9] А. О. Смирнов. Матем. заметки. 1995. Т. 58. № 1. С. 86-97.

[10] F. Gesztesy, R. Weikard. Bull. Amer. Math. Soc., New Ser. 1998. V. 35. № 4. P. 271-317.

[11] F. Gesztesy, H. Holden. Hierarchies of Soliton Equations and their Algebro-Geometric Solutions. V. 1. In: Cambridge Studies in Advanced Mathematics. Cambridge: Cambridge Univ. Press, 2002. (in press).

[12] F. Gesztesy, R. Ratnaseelan. Rev. Math. Phys. 1998. V. 10. P. 345-391.

[13] D. V. Chudnovsky. J. Math. Phys. 1979. V. 20. № 12. P. 2416-2422.

[14] Yu. V. Brezhnev. Rep. Math. Phys. 2001. V. 48. № 1/2. P. 39-46.

[15] M. Blaszak. Multi-Hamiltonian Theory of Dynamical Systems. Berlin: Springer, 1998.

[16] И. М. Гельфанд, Л. А. Дикий. Функц. анализ и его прилож. 1979. Т. 13. № 1. С. 8-20.

[17] В. З. Энольский. ДАН СССР. 1984. Т. 278. № 2. С. 305-308.

[18] M. Blaszak. Rep. Math. Phys. 2000. V. 46. № 1/2. P. 35-46.

[19] Н. В. Устинов, Ю. В. Брежнев. УМН. 2002. Т. 57. № 1. С. 167-168; nlin.SI/0012039.

[20] Ю. В. Брежнев. УМН. 2002. Т. 57. № 2. С. 191-192; nlin.SI/0106024. 